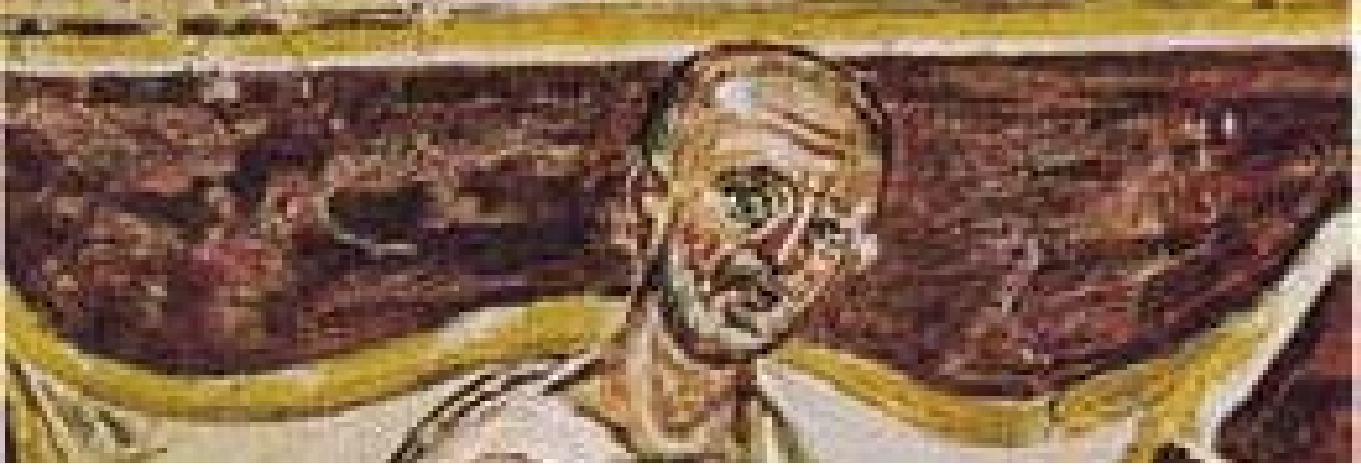

\section{Tres demostraciones de la existencia de Dios a partir de los Soliloquios de San Agustín}

Three demonstrations of the existence of God from the Soliloquios of San Agustin

\section{Resumen}

En este trabajo hago explícitas y analizo tres demostraciones de la existencia de Dios, implícitas en la meditación sobre la noción de verdad que se encuentra en los Soliloquios de San Agustín. La primera es a partir de la inteligibilidad de los seres. La segunda, a partir de las características ontológicas de las esencias inteligibles. La tercera la propongo aquí tomando inspiración de la aporía, que el autor analiza en el segundo libro de la mencionada obra, al intentar definir la verdad. La aporía emergente del análisis deriva de la observación que no es posible definir el concepto de verdad sin relación con un sujeto, pero tampoco es posible definirla con relación a un sujeto meramente temporal y contingente. De ahí, argumento la necesidad de postular la existencia de un Sujeto necesario y absoluto, que permita cumplir la definición de la verdad de un modo que no incurra en contradicción.

\section{Palabras clave}

Agustín de Hipona; Soliloquios; definición de la verdad; existencia de Dios.

\section{Abstract}

In this work I make explicit and analyze three demonstrations of the existence of God, that are implicit in the meditation on the notion of Truth in Augustine's Soliloquies. The first is from the intelligibility of beings. The second, from the ontological characteristics of intelligible essences. The third, which I propose, takes inspiration from the aporia that the author analyzes in the second book of the aforementioned work, when trying to define truth. The aporia emerging of the analysis is due to the observation that it is not possible to define the concept of truth without relation to a subject, but it is also not possible to define it in relation to a merely temporal and contingent subject. Hence, I argue the need to postulate the existence of a necessary and absolute Subject, which allows the definition of truth in a way that does not incur contradiction.
\end{abstract}

\section{Keywords}

Augustine of Hippo; Soliloquies; Definition of Truth; Existence of God.

\section{CLAUDIO PIERANTONI \\ Universidad de Chile, Chile Universidad de los Andes, Chile}

Claudio Pierantoni, Doctor en Historia del Cristianismo (1996, Università La Sapienza, Roma); Doctor en Filosofía (2017, Universidad de Los Andes, Santiago de Chile). Profesor de Historia de la Iglesia Antigua y Patrología en la Facultad de Teología de la P. Universidad Católica de Chile (Bachillerato y Magister en Teología) desde 2000 hasta 2010. Profesor de Filosofía Medieval en la Facultad de Filosofía de la Universidad de Chile (desde 2011 hasta el presente). Profesor de Literatura Latina y Monográfico de Dante en la Facultad de Literatura de la Universidad de Los Andes (desde 2012 hasta el presente).

Entre las últimas publicaciones, se destaca: Pierantoni, Claudio: Una Veritas. The Inclusive Definition of Truth as a Proof for the Existence of God, Editiones Scholasticae, Neunkirchen-Seelscheid, 2018.

\section{ORCID \#}

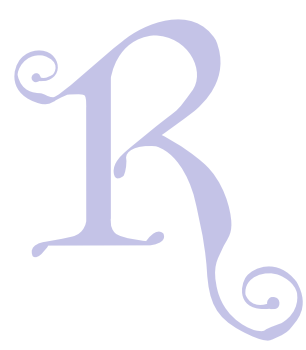

Recepción de artículo: 22-5-2019

Aceptación del artículo: 15-1-2020 


\section{CONSIDERACIONES INTRODUCTORIAS.}

Los Soliloquios, como es sabido, son uno de los primeros cuatro diálogos escritos por Agustín, reelaborando las conversaciones y meditaciones tenidas con amigos y familiares, en el tranquilo retiro de la villa de Cassiciacum, durante su preparación al bautismo, en la primavera del año $387 .^{1}$

Característica peculiar de este escrito, sin embargo, es que se trata de una conversación "a solas", como lo dice el título. A pesar de eso, no sería exacto afirmar que aquí Agustín "habla consigo mismo": en cambio, el joven converso, y apasionado filósofo, debate "con su propia Razón". La Razón es, en efecto, la facultad con la cual el ser humano puede descubrir y entrar en contacto con la verdad. Ahora bien, la verdad, que habita en el interior del hombre ('in interiore homine'), como dirá en el De vera religione, no por eso es una "parte" del ser humano, o una emanación de él. Muy por el contrario, la verdad se descubre como situada "frente" al hombre, como centro de una irreductible e inmutable objetividad. La verdad, que se descubre en la interioridad, es el centro de gravedad para el hombre y, en definitiva, lo es para toda la realidad.

Puede decirse que el tema de la verdad es el tema central de los Soliloquios agustinianos, a pesar de que esto no coincide formalmente con el tema anunciado al comienzo de la obra, inmediatamente después de la larga y bellísima invocación a Dios que ocupa todo el primer capítulo. Al comienzo del segundo capítulo, a la pregunta de la Razón: '¿Qué cosa, entonces, quieres conocer?', Agustín responde: 'Quiero conocer a Dios y al alma.' ¿Nada más?' 'Nada en absoluto', responde Agustín. Sin embargo, el joven filósofo es consciente de que no podrá afrontar estos temas sin primero examinar el problema de la verdad:

$\mathrm{R}$ ¿Entonces, no quieres conocer la verdad?

A. ¡Como si pudiera conocer estas cosas si no fuera a través de ella!

R. Entonces, primero hay que conocer a ella, por medio de la cual pueden conocerse estas cosas. ${ }^{3}$

Podrían meditar atentamente estas palabras todos aquellos filósofos que, a partir de Kant, aplican al pensamiento antiguo y medieval, bastante a la ligera, la etiqueta de "realismo ingenuo", o "realismo dogmático"; como si Kant realmente hubiese descubierto algo nuevo al afirmar que la razón no puede aventurarse a afirmaciones de carácter metafísico sin primero examinarse a sí misma. En efecto, como seguiremos viendo en esta presentación, Agustín es plenamente consciente de que es precisamente en un examen riguroso de la razón misma y de su relación con la verdad, que se basan las más importantes afirmaciones metafísicas. $E$, inversamente, es precisamente en el error en el planteamiento de la relación entre la razón humana y la verdad donde se originan los más profundos desaciertos en la metafísica.

Digamos en seguida que no se encuentran en los Soliloquios, propiamente hablando, demostraciones de la existencia de Dios que sean anunciadas de manera explícita, ni desarrolladas formalmente. Se trata más bien de una serie de elementos que Agustín proporciona y que pueden ser desarrollados y utilizados para formular demostraciones completas. ${ }^{4}$ En el caso de la tercera, como veremos, no se trata, propiamente hablando, de una demostración, sino más bien de un largo análisis de una aporía: esta aporía es la que será utilizada como base para realizar una demostración per absurdum. Es por este motivo que, en el título de este trabajo, he hablado de demostraciones "a partir de" los Soliloquios agustinianos. Los elementos nuevos agregados por mí al desarrollo agustiniano son evidentemente de mayor peso en la tercera de estas demostraciones: es por eso que le he dedicado una mayor extensión en la economía de este trabajo, y he sentido la necesidad de completarla con un debate con posturas nuestras contemporáneas acerca de la noción de verdad.

Después de estas consideraciones introductorias, pasemos ahora directamente a considerar nuestro tema.

\section{LA PRIMERA DEMOSTRACIÓN (A PARTIR DE LA INTELIGIBILIDAD DE LOS SERES)}

En la primera demostración que tomaremos en consideración, Agustín parte cuestionando qué tipo de método sería necesario utilizar para investigar acerca de la naturaleza divina. Empieza descartando un tipo de investigación de carácter empírico, dando por supuesto que Dios no es un ente que cae bajo la experiencia de los sentidos corporales. Dios pertenece a aquella categoría de entidades 'que se alcanzan mediante el intelecto'. ${ }^{5}$ En otras palabras, se trata de un Ser que se alcanza con el puro intelecto. Esto, aunque aparentemente dificulta la investigación, por otro lado resulta ser un elemento a favor: si bien los entes sensibles (por ejemplo, el amigo Alipio, la luna, o la cena de Agustín), parecen estar más al alcance del conocimiento humano, en realidad la ciencia que se refiere a ellos no es "ciencia" en el sentido estricto, pues no tiene el carácter de la necesidad. ${ }^{6}$ Por ejemplo, Agustín no está seguro de lo que comerá mañana para la cena; por otro lado Dios, o alguna oculta causa natural, podría improvisamente alterar el curso de la luna.

En segundo lugar, tampoco es posible, propiamente hablando, "saber" acerca de Dios sobre la base de la autoridad de otros filósofos, por ejemplo, Platón o Plotino, no solo porque ellos podrían haberse equivocado, al menos en parte, sino sobre todo porque en el caso de un Ser puramente inteligible no es suficiente la "creencia" que, aun

1. Véase Simonetti 1992, pp. xiii-xxviii.

2. R. Quis ergo scire vis? A. Deum et animam scire cupio. R. Nihilne plus? A. Nihil omnino. (Soliloquia l,ii, 7). Todas las traducciones que presento son mías.

3. Soliloquia l, ii, 7

4. También debe observarse el hecho que, en general, Agustín no está directamente interesado tanto en demostrar la existencia de Dios, cuanto en mostrar las características de la esencia divina frente a errores y deformaciones. En otras palabras, los interlocutores o adversarios a quienes se dirige no son tanto los que niegan la existencia de Dios, sino los que le atribuyen características equivocadas.

5. Ex eo sunt genere quae intellectus assequitur (Soliloquia l,iii, 8)

6. Cf. Platón, Timeo 28a.29c. 
cuando es verdadera, es siempre indirecta, es decir, basada sobre el crédito que le concedemos a otra persona. En otras palabras, en el caso de Dios debemos aspirar a un conocimiento directo de Él: lo que es, propiamente hablando, la "ciencia".

En cuanto Ser puramente inteligible, prosigue la Razón, Dios puede ser considerado en analogía con los entes matemáticos, cuya verdad necesaria es directamente accesible a nuestro intelecto. Agustín protesta que Dios es inmensamente superior a tales entidades. Sin embargo, observa la Razón, aquí no está en cuestión el grado o nivel ontológico de Dios, sino (a) el tipo de conocimiento que es posible acerca de Él y (b) el grado de certeza al que podemos aspirar en dicho conocimiento. Haciendo una analogía con la percepción sensible, podemos decir que, desde un punto de vista epistemológico, el tipo de conocimiento que nos proporcionan los sentidos no depende del grado de valor que atribuimos a cada ente sensible.

Si examinamos los entes matemáticos, lo que se observa es que su elaboración necesita basarse también en la experiencia sensible: ésta, con una sugerente imagen, es comparada a un barco que, después de habernos transbordado a través de los continuos movimientos del "oleaje" (es decir, del mundo sensible, en continuo cambio), finalmente toca "tierra": o sea, nos hace desembarcar en la tierra firme del conocimiento puramente inteligible (que es inmutable).

En esta "tierra", naturalmente, podemos fácilmente reconocer el "mundo real" del relato platónico de la caverna: el mundo de los "verdaderos seres". ${ }^{7}$ Aquí queda clara la analogía, aunque también la infinita distancia que separa los entes humanamente inteligibles -como los entes matemáticos- del Ser divino trascendente. Escribe Agustín:

La Razón que te habla mostrará Dios ante tu mente, del mismo modo en que el sol se muestra a los ojos. En efecto, la mente para el alma es como su sentido: y todas las afirmaciones más ciertas de las ciencias son tales cuales aquellas cosas que son iluminadas por el sol, para que puedan ser vistas, tal como la tierra y todas las cosas terrenas: Dios mismo, por su parte, es quien las ilumina. Yo, en cambio, la Razón, soy en la mente aquello que en los ojos es la vista. ${ }^{8}$

Podemos deducir de esta analogía al menos dos conceptos fundamentales, explicitando lo que Agustín sugiere implícitamente:

1. Así como no es posible (o muy difícil) a los ojos materiales mirar directamente al sol, por su excesiva luminosidad física, tampoco es posible a la mente (salvo en un nivel sobrenatural) mirar directamente al Sol inteligible, Dios.

2. Pero, así como el sol sensible debe ser postulado como causa de la visibilidad de los objetos materiales, de la misma manera debe postularse el Sol inteligible como causa de la inteligibilidad de todos los objetos inteligibles. O, mejor dicho, como causa de la misma existencia de los objetos inteligibles.
3. Por lo tanto, se impone necesariamente la siguiente consecuencia: el conocimiento de la existencia del Sol inteligible es tan cierto como el del más cierto de los inteligibles.

Recibe de mi enseñanza, ahora, por cuanto lo exige la circunstancia presente, de aquella similitud de los entes sensibles, también algo de Dios. En efecto, Dios es inteligible, y también aquellos elementos de la ciencia; sin embargo, son muy diferentes. Pues, también, la tierra es visible, como también la luz; pero la tierra, si no es iluminada por la luz, no se puede ver. Por tanto, también aquellas cosas que se enseñan en las disciplinas (que, cualquiera que las entienda, concede que son, sin duda alguna, absolutamente verdaderas), hay que creer que no podrían entenderse, si no fueran iluminadas por algún sol suyo propio. Así, tal como en este sol se pueden notar tres cosas: que existe, que resplandece y que ilumina, así también, en ese Dios secretísimo, que tú quieres entender, hay tres cosas: que existe, que se entiende, y que hace entender las demás cosas. Estas dos cosas, es decir, ti mismo y Dios, me atreveré a enseñártelas, para que las entiendas. ${ }^{9}$

Por un lado, vemos que en este pasaje está clara la infinita diferencia cualitativa entre el Sol que es Dios y los inteligibles humanos que nos son directamente accesibles. La inteligibilidad de estos, en efecto, resulta ser derivativa: ellos deben fundarse en una "fuente de lo inteligible", que no podemos contemplar de modo directo. Por otro lado, resulta claro que, sin postular la existencia de esta fuente, inmutable y necesaria, no se podría concebir una causa suficiente de la certeza de los principios de las ciencias, o, para decirlo mejor, del primer principio de la ciencia en general. Por lo tanto, resulta probado que la existencia de Dios nos es conocida con una ciencia tan cierta como la ciencia de los primeros principios de la mente.

\section{LA SEGUNDA DEMOSTRACIÓN (A PARTIR DE LAS CARACTERÍSTICAS ONTOLÓGICAS DE LAS ESENCIAS INTELIGIBLES)}

Pasemos ahora a la segunda demostración, que se encuentra un poco más adelante en el capítulo xv, el último del libro I. A diferencia de la anterior, que partía considerando los entes matemáticos, esta se basa en la predicación de "lo verdadero", hecha a propósito de los entes contingentes. Consideremos la diferencia: decir que 'un teorema de matemática es verdadero', no es lo mismo que decir 'un árbol es verdadero'. En efecto, la esencia ideal de un teorema de matemática tiene su propia autonomía inmutable, independiente de toda esencia real. En cambio, la esencia ideal de un árbol, su "verdad" de árbol, está en relación con su sustancia real; sin embargo, no coincide con ella, es decir, con la existencia de un árbol en el mundo real. Si bien es cierto que la verdad del árbol se predica de "este" árbol, cuando decimos "este es un verdadero árbol", eso no significa que la verdad del árbol (su esencia ideal) se circunscriba a este árbol particular. Pues, por un lado, ella se predica igualmente de todos los demás árboles; por otro lado, la esencia ideal queda intacta al morir este árbol, incluso

7. Cf. Platón, República VII, 514a-518 d.

8. Promittit enim ratio quae tecum loquitur, ita se demonstraturam Deum tuae menti, ut oculis sol demonstratur. Nam mentes quasi sui sunt sensus animis; disciplinarum autem quaeque certissima talia sunt, qualia illa quae sole illustrantur, ut videri possint, veluti terra est atque terrena omnia: Deus autem est ipse qui illustrat. Ego autem ratio ita sum in mentibus, ut in oculis est aspectus. (Soliloquia I,vi, 12)

9. Nunc accipe, quantum praesens tempus exposcit, ex illa similitudine sensibilium etiam de Deo aliquid nunc me docente. Intellegibilis nempe Deus est, 
si desapareciesen todos los árboles del mundo. Debe concluirse, sigue Agustín, que la verdad no se predica del árbol 'como un accidente en un sujeto': pues de otro modo, al desaparecer el sujeto, desaparecería también el accidente. Pero, al mismo tiempo, Agustín se da cuenta de que la verdad tampoco puede existir independientemente de todo sujeto, es decir, de toda realidad. Es por eso, que debe postularse la existencia de una realidad estable y eterna, un "lugar metafísico", por así decirlo, que fundamente ontológicamente la existencia de la verdad, en cuando ser puramente ideal. Aquella realidad eterna, podemos concluir, es lo que llamamos Dios.

En una exposición algo más analítica de esta demostración, podemos distinguir los siguientes cuatro pasos.

El primer paso consiste en distinguir "la verdad" de "lo verdadero". La verdad es el concepto general, que, como tal, es lógicamente anterior a los entes particulares de los cuales se predica. Así como, por ejemplo, la castidad hace al casto, del mismo modo la verdad "hace" lo que es verdadero. Dicho de otro modo, el que es casto lo es por la castidad: así, lo que llamamos verdadero lo es por la verdad. En la terminología platónica, lo verdadero es tal, porque "participa" de la verdad. No se podría llamar algo "verdadero", sin hacer previa, aunque sea implícita, referencia a la "verdad". La "verdad" como tal, por lo tanto, es lógica y ontológicamente anterior a "lo verdadero" (en el sentido de "algo verdadero" en particular).

El segundo paso, más importante aún, consiste en mostrar cómo "lo verdadero" puede extinguirse, o morir, pero no así la verdad. Por ejemplo, un árbol es sin duda "algo verdadero" (un verdadero árbol), que por supuesto nace y muere. Sin embargo, "la verdad del árbol" no puede morir, ni tampoco - agregamos - puede cambiar en absoluto. La verdad del árbol, en efecto, como pura esencia inteligible que es, no se ve afectada por la existencia real del árbol (que no es necesaria, sino contingente). Así, se ha averiguado que la verdad existe en un plano ontológico diferente, el plano de lo inmutable, netamente distinto del plano temporal o histórico.

Tercer paso: debe suponerse, por lo tanto, que tiene que haber algún "lugar" (o substantia) donde se coloca la verdad, que no es un lugar de este universo en sentido físico, pero que, sin embargo, debe ser algo real. Verdadera es la sentencia que dice: 'quidquid est, alicubi esse cogitur' ('todo lo que es, es necesario que esté en algún lugar')10. Como se ve, el texto original, con el adverbio alicubi, evita mencionar la palabra "lugar", que en castellano estamos obligados a introducir. Esto le permite a Agustín precisar a continuación, justamente, que no se trata de un "lugar" (sobreentendiendo, "en el sentido ordinario" )11.
Por lo tanto, es necesario que investiguemos dónde está: pues no está en un lugar, a no ser que acaso pienses que algo más allá del cuerpo exista en un lugar, o que la verdad sea un cuerpo. ${ }^{12}$

En otras palabras, se necesita encontrar un "lugar metafísico", donde colocar la verdad, para que nadie piense que deba colocarse en un lugar físico, dado que en este solo podría colocarse una realidad corpórea. No debes pensar - dice la Razón - que la verdad sea un cuerpo. Ciertamente, este lugar no debe buscarse entre las cosas mortales ('in rebus mortalibus'), pues ya hemos visto que ella no muere; por tanto, también debe ser inmortal el "lugar" donde ella se ubica:

Por tanto, la verdad no está en las cosas mortales. Y sin embargo la verdad existe y está en algún lugar: por lo tanto, existen realidades inmortales. ${ }^{13}$

El silogismo es breve y concluyente. Desarrollándolo un poco con una terminología más familiar para nosotros, podemos expresarlo así:

1. Las esencias ideales existen y son inmutables.

2. Siendo realidades puramente virtuales (conceptuales), ellas no pueden existir absolutamente por sí mismas: deben tener un "lugar de residencia metafísica", por así decirlo, que sea real, que constituya el "soporte" sustancial sin el cual ellas no podrían existir, justamente porque son esencias meramente conceptuales.

3. Por lo tanto, existe una realidad imperecedera, que es a la que se llama Dios.

Nótese que Agustín tiene plena conciencia de que la "verdad" puramente conceptual no debe ser concebida por sí misma, como metafísicamente subsistente. Dado que no es substantia, ella existe necesariamente "en" una sustancia. Pero es claro que ella no puede residir en una sustancia contingente al modo de un accidente: pues la "verdad de algo" no es, evidentemente, un atributo que se agregue a la sustancia y que dependa de ella para existir, y pueda perecer con ella, sino que es la esencia inteligible de la sustancia misma, y, como tal, preexiste al ente contingente. Precisamente por eso, debe existir una realidad eterna, que preexiste a la realidad temporal y la funda causalmente.

Si bien aquí no se menciona explícitamente el nombre de Dios, este 'pequeño razonamiento' (ratiuncula) es una concluyente demostración de su existencia, a partir de la verdad inmutable de las esencias ideales.

intellegibilia etiam illa disciplinarum spectamina; tamen plurimum differunt. Nam et terra visibilis, et lux; sed terra, nisi luce illustrata, videri non potest. Ergo et illa quae in disciplinis traduntur, quae quisquis intellegit, verissima esse nulla dubitatione concedit, credendum est ea non posse intellegi, nisi ab alio quasi suo sole illustrentur. Ergo quomodo in hoc sole tria quaedam licet animadvertere; quod est, quod fulget, quod illuminat: ita in illo secretissimo Deo quem vis intellegere, tria quaedam sunt; quod est, quod intellegitur, et quod caetera facit intellegi. Haec duo, id est, teipsum et Deum, ut intellegas, docere te audeo. (Soliloquia I,viii, 15)

10. Soliloquia I, Xv,29, al comienzo.

11. 'Aliter, non quasi in loco' se dice en las Confesiones, en un texto notable: Et respexi alia et vidi tibi debere quia sunt et in te cuncta finita, sed aliter, non quasi in loco, sed quia tu es omnitenens manu veritate, et omnia vera sunt, in quantum sunt. (Conf. VII, XV,21)

12. Soliloquia I,xv,29: Ergo ubi sit, necesse est quaeramus; non est enim in loco, nisi forte aut esse in loco aliquid aliud praeter corpus, aut veritatem corpus esse arbitraris.

13. Soliloquia I,xv,29: Non igitur est veritas in rebus mortalibus. Est autem veritas et non est nusquam. Sunt igitur res immortales. 


\section{LA TERCERA DEMOSTRACIÓN (A PARTIR DE LA APORÍA EN LAS DEFINICIONES DE LA VERDAD)}

La tercera demostración, como lo hemos anticipado, en realidad no nos la proporciona directamente Agustín. Más bien, el autor pone de manifiesto la aporía a partir de la cual el que quiera puede desarrollar una demostración que podemos llamar per absurdum.

Esta aporía se desarrolla a lo largo de la gran parte del libro II. Después de la conocida demostración de la eternidad de la verdad, que ocupa los primeros capítulos del libro, Agustín se concentra en el intento de definir la verdad: 'Quid est veritas?' A partir de esta pregunta, que no es otra sino la pregunta de Pilato en el Evangelio según san Juan, ${ }^{14}$ se desarrollará una parte central de toda la filosofía occidental.

Agustín parte definiendo la verdad, o más exactamente 'lo verdadero' (verum) como 'aquello que es tal como parece'. Esta definición es aparentemente intachable. Pero se presenta un problema: en efecto, decir que lo verdadero es aquello que es "tal como parece", supone que haya siempre "alguien" a quien le parece. Y esto, evidentemente, no es siempre el caso: pues, suponiendo por ejemplo que los seres humanos dejaran de existir, entonces 'los árboles no serían verdaderos árboles'. Incluso en este momento, dice la Razón, las piedras ocultas que se hallan en el fondo del océano, donde nadie tiene acceso, no serían verdaderas piedras, porque no son conocidas por ningún ser humano. Es más, dichas piedras tampoco podrían ser verdaderos entes: en otras palabras, simplemente no serían.

Para evitar este grave problema, un verdadero naufragium, Agustín recurre a una definición que elimine el sujeto y se concentre en el ser de la cosa. Según esta segunda definición, 'verdadero es lo que es'.

Esta definición ciertamente viene a salvar a Agustín (y a nosotros) del peligro de que piedras, árboles y todos los seres del mundo se nos desvanezcan en la pura nada. Sin embargo, la Razón no tarda en hacerle notar que, en este caso, también 'desaparecería lo falso'. Es decir, todos los entes, en cuanto tales, serían sin duda verdaderos, pero perderían todo significado para nosotros y para nuestra búsqueda, que precisamente se esfuerza por investigar la verdad a través de la diferencia con la falsedad. Dicho de otro modo, eliminar al sujeto (humano) de la definición de la verdad, mientras por un lado parece salvaguardar la entidad de las cosas en sí, por otro lado, no da razón de su relación como cosas relevantes para nosotros. En esta definición, en efecto, las cosas resultan en sí mismas existentes, pero sin relación con su inteligibilidad "para alguien". De este modo, "ser verdadero" sería simplemente sinónimo de "ser", por lo tanto, no agregaría nada al puro concepto del ser. ${ }^{15}$
Para comprender mejor este punto, puede introducirse aquí una breve referencia a la teoría contemporánea de la verdad como "identidad", que ha sido propuesta como alternativa a la definición tradicional de la verdad como 'adaequatio intellectus et rei'. En esta línea ha sido argumentado, en tiempos más cercanos a nosotros, que el considerar la verdad como algo distinto del ser de los hechos sería una "redundancia". Es decir, cuando, por ejemplo, se afirma que 'Sócrates es sabio', se diría lo mismo que cuando se afirma 'es verdad que Sócrates es sabio'. Bastaría con quitar la frase de la "cita" que la contiene, precedida por la introducción "es verdad que" y tendríamos exactamente el mismo contenido; de manera que la locución "es verdad que" sería superflua. ${ }^{16}$

En esencia, según esta teoría, se da una identidad entre el "contenido" de una proposición y el "hecho" que tal proposición expresa. De este modo, se busca dar al lenguaje un contenido directamente constituido por "Ios hechos mismos" que evite el peligro, que algunos perciben en la teoría de la verdad como correspondencia, de que ya no sea posible establecer un puente entre el contenido del pensamiento y los hechos, una vez que se establezca su distinción. A comienzos del siglo XX, así se expresa por ejemplo Moore:

Una vez que se reconoce definitivamente que la proposición es denotar no una creencia o una forma de palabras, sino un objeto de creencia, parece claro que una verdad no difiere en absoluto de la realidad con la que se supone que debe corresponder. ${ }^{17}$

Explica Gaskin:

El significado de la teoría de la identidad, para sus partidarios, es que parece proporcionar el cierre de una cierta brecha que de otra manera podría pensarse que se abre entre el lenguaje y el mundo y/o entre la mente y el mundo.' ${ }^{\prime 8}$

Y más adelante:

El problema parece no estar relacionado con la existencia de tales hechos, sino más bien con la relación de correspondencia que la versión de la teoría de la correspondencia que actualmente estamos considerando afirma que se da entre el hecho de que Sócrates es sabio y la proposición que Sócrates es sabio. Tal como se desprende de esta manera de expresar la dificultad, no parece haber diferencia lingüística entre la forma en que hablamos sobre las proposiciones y la manera en que hablamos sobre los hechos, cuando estas entidades están especificadas por las cláusulas "que". Esto sugiere que los hechos solo son proposiciones verdaderas. Si eso es correcto, entonces la relación entre los hechos y las proposiciones verdaderas no es de correspondencia, lo que, como Frege observó famosamente (cf. Frege

14. In 18,38 .

15. La misma aporía será señalada por Tomás de Aquino: Videtur autem quod verum sit omnino idem quod est. [...] Sed contra: Nugatio est eiusdem inutilis repetitio. Si ergo verum esset idem quod ens, esset nugatio, dum dicitur 'ens verum': quod falsum est. Ergo non sunt idem. (Quaestio / de veritate, art. 1).

16. Ésta ha sido también llamada, por tal motivo, 'disquotational theory of Truth' o también 'deflationary theory of Truth'. (cf. Gaskin 2015).

17. Once it is definitely recognized that the proposition is to denote not a belief or a form of words, but an object of belief, it seems plain that a truth differs in no respect from the reality with which it was supposed merely to correspond. (Moore 1901-2, p.717). También Bertrand Russell, que en su periodo temprano defendió una variante de la teoría de la correspondencia, posteriormente, influido por Moore, aceptó una versión de la teoría de la identidad (Russell 1903).

18. The significance of the identity theory, for its supporters, is that it appears to make available the closing of a certain gap that might otherwise be thought to open up between language and world and/or between mind and world. (Gaskin 2015) 
1918-19, p. 60), implica la distinción de los relata, sino de identidad. ${ }^{19}$

Resumiendo: el punto fuerte de esta postura es el siguiente: no hay diferencia lingüística entre el modo en que hablamos de las proposiciones ("es verdad que") y el modo en que hablamos de los hechos ("es el caso que"). Esto, innegablemente, es así: y permite iluminar la primera definición que Agustín nos da de la verdad: 'verdadero es lo que es'. Es decir, "en la verdad", el hecho es precisamente el que es. Si, al hablar, el hecho del que hablamos no fuera precisamente "ese hecho", es claro que no podríamos estar hablando de ese hecho: éste, por la hipótesis, no estaría "en nuestro pensamiento". Los hechos, necesariamente, nos son accesibles a través de nuestro pensamiento. En este sentido, es verdad que el hecho "presentado" por nuestro concepto es el hecho mismo. De otro modo, estaríamos negando que tenemos acceso a los hechos mismos. Con todo -y aquí está el error en que cae esta teoría- el que aquello que es presentado a nuestro pensamiento por medio del concepto sea el hecho mismo, no significa que el concepto, es decir aquel "vehículo" o "medio" que nos presenta la realidad ('illud quo', en la terminología escolástica), sea idéntico a la realidad misma que conocemos ('illud quod'). Eso equivaldría a sostener que la realidad se nos manifiesta de modo directo, sin mediaciones: pero, si así fuera, nuestros conceptos serían siempre absolutamente verdaderos, pues serían los hechos mismos presentes en nuestra mente absolutamente hablando, sin limitación ni error: en palabras de Agustín, 'no existiría lo falso'. En contra, es evidente que lo falso para nosotros sí existe: que nuestros conceptos son limitados, muchas veces erróneos, incluso contradictorios entre ellos. Si los conceptos en nuestra mente fueran directamente "los hechos", entonces habría que admitir que los hechos mismos podrían ser contradictorios consigo mismos: la realidad misma sería auto-contradictoria, lo cual es absurdo. Además, puede demostrarse que los conceptos son siempre idénticos a sí mismos, inmutables, a diferencia de los hechos, que cambian, son temporales. Es por eso que ya los griegos, o al menos Platón y Aristóteles (muchos siglos antes que Frege), habían subrayado que una correcta teoría de la verdad implica la distinción de los relata (proposición y realidad).

Volviendo ahora al texto de los Soliloquios, observamos que el análisis sigue, complicándose un poco en diferentes definiciones de lo falso, que no será necesario analizar aquí. Lo esencial que notamos es que, de diferentes maneras, vuelve a plantearse la necesidad de reintroducir al sujeto en la definición de la verdad. Pero constantemente se vuelve a producir la misma aporía de que, si por un lado se necesita esencialmente un sujeto para definir la verdad, por otro lado, el sujeto humano, con su límite y contingencia, no cumple con las características ontológicas necesarias para garantizar la esencial verdad de las cosas, que permanece tal cual en ausencia del sujeto contingente.

De ahí que es posible deducir la única salida para esta aporía. Esta deducción, como decíamos, no la realiza Agustín, dejando así para nosotros la tarea. A estas alturas, no es difícil sacar la conclusión: pues la única salida posible de la aporía que hemos analizado siguiendo a Agustín, es postular que el Sujeto que se requiere para poder cumplir la definición de la verdad es un intelecto esencialmente existente, que efectivamente permita que se cumpla una característica necesaria de todos los seres, es decir, la de ser inteligibles: sin una intrínseca inteligibilidad, los seres no serían concebibles. Esto implica una necesaria relación con un intelecto, y por lo tanto la existencia real de un Intelecto que sea absoluto, necesario - netamente distinto del nuestro: el Intelecto divino.

En conclusión, dado que la definición de la verdad es ella misma esencial y necesaria, en su nivel de existencia puramente objetiva, es necesario postular la existencia real de un Sujeto absoluto que permita cumplirla, sin el cual la definición misma de la verdad resultaría contradictoria. ${ }^{20}$

19. The problem would seem to be not with the existence of such facts, but rather with the relation of correspondence which is said by the version of the correspondence theory that we are currently considering to obtain between the fact "that Socrates is wise" and the proposition "that Socrates is wise". As emerges from this way of expressing the difficulty, there seems to be no linguistic difference between the way we talk about propositions and the way we talk about facts, when these entities are specified by "that" clauses. That suggests that facts just are true propositions. If that is right, then the relation between facts and true propositions is not one of correspondence-which, as Frege famously observed (cf. Frege 1918-19, p. 60), implies the distinctness of the relata-but identity.' (Gaskin 2015, cap. 3)

20. Puede verse un desarrollo y una fundamentación más completa de esta demostración de la existencia de Dios, con detallados análisis de otros autores y una amplia conclusión sistemática, en mi libro: Pierantoni, 2018. 
- Augustinus: Soliloquia, De immortalitate animae, De quantitate animae, ed. W. Hörmann, C.S.E.L. LXXXIX, Vindobonae 1986.

- Frege, Gottlob: “Der Gedanke: eine logische Untersuchung”, Beiträge zur Philosophie des deutschen Idealismus, 2, $1918-19$.

- Gaskin, Richard: "The Identity Theory of Truth", The Stanford Encyclopedia of Philosophy (Summer 2015 Edition), Edward N. Zalta (ed.)

- $\mathrm{URL}=$ http://plato.stanford.edu/archives/sum2015/entries/truth-identity/).

- Moore, George Edward: Truth and Falsity, 1901-02, reimpr. en: Selected Writings, Ed. T. Baldwin, London and New York 1993.

- Pierantoni, Claudio: Una Veritas. The Inclusive Definition of Truth as a Proof for the Existence of God, Editiones Scholasticae, NeunkirchenSeelscheid, 2018.

- Platonis: Opera, Tomus IV, Tetralogiam VIII continens, ed. J. Burnet, Clarendon, Oxford, 1962.

- Platonis: Rempublicam, ed. S.R. Slings, Clarendon, Oxford, 2003.

- Russell, Bertrand: The Principles of Mathematics, London 1903.

- Simonetti, Manlio, ed.: Sant'Agostino, Confessioni, Vol. I, Fondazione Lorenzo Valla, Roma 1992. 